DSF $25 / 2003$

astro-ph/0307213

\title{
Present status of primordial nucleosynthesis after WMAP: results from a new BBN code
}

\author{
A. Cuoco, F. Iocco, G. Mangano, G. Miele, O. Pisanti, and P.D. Serpico \\ Dipartimento di Scienze Fisiche, Universitá di Napoli "Federico II", and INFN, Sezione \\ di Napoli, Complesso Universitario di Monte Sant'Angelo, Via Cintia, I-80126 Napoli, \\ Italy
}

\begin{abstract}
We report on the status of primordial nucleosynthesis in light of recent results on CMB anisotropies from WMAP experiment. Theoretical estimates for nuclei abundances, along with the corresponding uncertainties, are evaluated using a new numerical code, where all nuclear rates usually considered have been updated using the most recent available data. Moreover, additional processes, neglected in previous calculations, have been included. The combined analysis of CMB and primordial nucleosynthesis prediction for Deuterium gives an effective number of relativistic degrees of freedom in good agreement with the simplest scenario of three non degenerate neutrinos. Our findings seem to point out possible systematics affecting ${ }^{4} \mathrm{He}$ mass fraction measurements, or the effect of exotic physics, like a slightly degenerate relic neutrino background.
\end{abstract}

PACS number(s): 98.80.Cq; 98.80.Ft 


\section{Introduction}

The recent results of WMAP collaboration on Cosmic Microwave Background (CMB) anisotropies [1] perhaps represent both a change of perspective and a further call for increasing precision in cosmology. Before their first data release, in fact, the determination of baryonic matter density $\omega_{b}=\Omega_{b} h^{2}$ was in the realm of Big Bang Nucleosynthesis $(\mathrm{BBN})$, providing a result which typically is a compromise between the values preferred by primordial Deuterium and ${ }^{4} \mathrm{He}$ experimental determinations (see for example [2]). The very accurate determination which is now available from CMB, $\omega_{b}=0.024 \pm 0.001$ [3] is an independent piece of information which may be used as a prior in BBN theoretical analysis. This has dramatic consequences in determining the internal consistency of the standard picture of light nuclei formation and/or the role of systematics in the experimental measurements. As we will discuss in the following, the Deuterium (density fraction with respect to $H)$ experimental result of [4, $X_{D}=\left(2.78_{-0.38}^{+0.44}\right) \cdot 10^{-5}$, is in quite a fair agreement with the theoretical prediction using the CMB value for $\omega_{b}$. This is a remarkable success of standard $\mathrm{BBN}$, since $X_{D}$ is a rapidly varying function of $\omega_{b}$. The status of the other two observed nuclei, ${ }^{4} \mathrm{He}$ and ${ }^{7} \mathrm{Li}$, is less satisfactory. The source for the tension between theory and experiments, though not so serious at the moment, may be due to systematics in the reconstruction of the primordial value from observations or rather to some more exotic features of the BBN theoretical scenario, as extra relativistic degrees of freedom or neutrino chemical potential.

To clarify all these issues it is therefore necessary at this stage to make an effort to further improve the accuracy of theoretical predictions, as well as to have new observation and measurement campaigns. In the recent few years some steps have been made in this direction: the accuracy of the neutron to proton ratio at decoupling [5]-[9] and the numerical stability of the original public BBN code [10] have been improved, the uncertainties of the theoretical results due to experimental errors on the several nuclear rates entering in the reaction network [1] have been quantified. This network, partially updated [12] with

the recent NACRE compilation [13], traces back to the original analysis of [14] and, more recently, of [15, 16]. In the last decade, however, many new experimental and more precise 
data have been obtained for relevant nuclear reactions in the energy range of interest for $\mathrm{BBN}$. It is therefore timely to perform a complete and critical review of the whole nuclear network, in order to refine the input values for the rates and, perhaps more crucial, to reduce the theoretical uncertainties on nuclide yields (mainly D and ${ }^{7} \mathrm{Li}$ ). In Section 2 we summarize the main results of such an analysis. All nuclear rates already present in the code of Wagoner-Kawano [10] have been updated using the most recent available data. Moreover additional processes, neglected in previous calculations, have been included in a new version of our BBN code. Relevant examples of updated reactions and new added ones are discussed in this paper, though a complete overview is in progress [17].

In the following we will be mainly interested in determining $\omega_{b}$ and the effective number of relativistic degrees of freedom, $N_{e f f}$, defined as

$$
\rho_{R}=\left[1+\frac{7}{8}\left(\frac{T_{\nu}}{T_{\gamma}}\right)^{4} N_{e f f}\right] \rho_{\gamma},
$$

with $\rho_{\gamma}$ the photon contribution to the total relativistic energy density, $\rho_{R}$. In the standard scenario three active massless, non degenerate, neutrinos are the only additional relativistic degrees of freedom. This results in $N_{\text {eff }}=3.04$ at the CMB epoch, which takes also into account the small entropy release to neutrinos during the $e^{+}-e^{-}$annihilation phase and QED plasma corrections [18]-[20]. This effect also slightly modifies BBN and results in an effective $N_{\text {eff }}=3.01$ for ${ }^{4} \mathrm{He}$ mass fraction [21, 22, 18]. As known from many analysis [23]-[28], if one considers $N_{\text {eff }}$ as a fitting parameter, CMB alone cannot severely constrain it, though WMAP data resulted in a sensible improvement of previous bound. We have performed a CMB likelihood analysis, described in Section 3, using the CMBFAST public code [29] and the WMAP software facilities to calculate the likelihood functions [30]-33]. Assuming that $N_{\text {eff }}$ is not changing between the BBN and CMB epochs, we show in Section 4 that a joint analysis of CMB data and Deuterium abundance nicely fits in the simplest standard scenario, with $N_{\text {eff }} \sim 3$.

As we mentioned, the present determination of ${ }^{4} \mathrm{He}$ mass fraction would require a slightly smaller value for $\omega_{b}$ than what is suggested by WMAP data. A possible way out to this discrepancy is of course to reduce the neutron to proton ratio at decoupling, e.g. to lower the relativistic energy density. It is therefore not a surprise that a purely 
BBN likelihood analysis of the $N_{e f f}-\omega_{b}$ parameters, described in Section 5, provides a preferred value for $N_{\text {eff }}$ smaller than three. We conservatively interpret this finding as a possible sign of systematics in the ${ }^{4} \mathrm{He}$ measurements. In Sections 4 and 5 the estimates of primordial abundances are discussed. Deuterium results for different quasars absorption systems (QAS) distribute around the theoretical expected value, while there is still an evidence at $2-3 \sigma$ level for primordial ${ }^{7} \mathrm{Li}$ depletion. We also report the theoretical prediction for ${ }^{6} \mathrm{Li}$ and ${ }^{3} \mathrm{He}$ abundances, which may represent further tests for BBN.

In Section 6 we perform an updated analysis of the degenerate BBN scenario. Similar studies are reported in [26, 34]. It has been shown in [35] that for large mixing angle oscillation solution to solar neutrino problem, chemical potentials of the three active neutrino species are much more severely bound than what was found in [23], since oscillations tend to homogenize the lepton asymmetries among the active neutrino flavors. In par-

ticular, the conservative bound on the asymmetry parameters, $\xi_{\alpha} \equiv \mu_{\nu_{\alpha}} / T_{\nu}$, quoted in [35] is $\left|\xi_{\mu, \tau}\right| \sim\left|\xi_{e}\right| \leq 0.07$. Of course there is still room for a small neutrino-antineutrino asymmetry in the universe, which may help in reconciling the slight discrepancy in the ${ }^{4} \mathrm{He}$ abundance. Remarkably, a tiny neutrino chemical potential has a great impact on the BBN favorite value for $N_{\text {eff }}$. We finally give our conclusions in Section 7 .

\section{A new code for $\mathrm{BBN}$}

In this Section we briefly describe the main aspects of a new BBN code realized starting from the original public version of Kawano [10]. This has been achieved in several steps, some being already described in previous papers [8, 9]. The main improvement is a complete review and update of all nuclear reaction rates which enter in the light nuclide network, as well as, more generally, a critical review of many other processes which were not considered in the standard BBN network. Some of these resulted to be not negligible and thus have been included for the first time. An exhaustive description of the analysis of all rates included in the code is in progress [17]. Here we only stress the key role of some of the processes we have either updated using new experimental results or included for the first time.

The main aspects of the new code can be summarized as follows: 


\section{1) Numerical features}

As discussed in [9, numerical resolution of differential equation describing the time evolution of nuclide abundance is quite critical. This is because the problem is stiff, since the right hand sides of corresponding Boltzmann equations, being the difference of almost equal forward and reverse process rates, are typically a small difference of two large numbers. To deal with this problem our code adopts a NAG routine [36] implementing a method belonging to the class of Backward Differentiation Formulae, instead of a traditional Runge-Kutta solver.

2) $n \leftrightarrow p$ weak rates and ${ }^{4}$ He mass fraction

The weak rates converting neutrons and protons are the key inputs deciding for the $n / p$ ratio at freeze-out and eventually for the ${ }^{4} H e$ mass fraction. The theoretical estimate for these rates was improved by considering QED radiative and finite temperature effects as well as finite nucleon mass [5]- [9], which result in a reduced uncertainty in ${ }^{4} \mathrm{He}$ mass fraction $Y_{p}$ at the level of $0.1 \%$. Present statistical (and possibly systematics) uncertainty of the $Y_{p}$ experimental determination, quoted at the level of $1 \%$, does not presently suggest any further effort in theoretical analysis of this issue. We have also updated the value of the neutron lifetime, $\tau_{n}=(885.7 \pm 0.8) s[37$.

3) An updated nuclear reaction network

The nuclear reactions involved in BBN form quite a complicated network of order hundred different processes. The original compilation [14] (standard network) was reviewed by Smith, Kawano and Malaney [15]. The standard chain was partially reanalyzed in [12] (for a more recent analysis see Ref. [16]) by extensively using the NACRE nuclear rate collection. Since then new data became available, as for example the LUNA collaboration results for $\mathrm{D}+p \rightarrow \gamma+{ }^{3} \mathrm{He}$ (see later) [38. It is well known that the uncertainties on several nuclear rates still represent the main limitation to any theoretical analysis and prediction of BBN. On the other hand, an improved precision of BBN theoretical estimates is mandatory, both to check the consistency of the physical grounds of BBN and to constraint the values of cosmological parameters. This can be achieved by a new complete study of all reaction rate network. Our analysis can be summarized as follows:

i) all two body strong and electromagnetic reactions entering in the production/destru- 
ction of light nuclei up to $A=7$ have been all reevaluated, using NACRE estimates for rates and errors. In particular the key reaction $n+p \leftrightarrow \gamma+D$ has been studied in details, both in its experimental and theoretical aspects, since it represents the main $D$ production process, therefore influencing all the heavier nuclide primordial production.

ii) for all processes not included in NACRE catalogue we have either used other available experimental data or simple theoretical models to get a reasonable estimate for the rates.

iii) new processes are now included in the code, as for example the ${ }^{7} \mathrm{Li}+{ }^{3} \mathrm{H} \leftrightarrow n+{ }^{9} \mathrm{Be}$ stripping/pickup reaction [39, and the analogous ${ }^{7} \mathrm{Li}+{ }^{3} \mathrm{He} \leftrightarrow p+{ }^{9} \mathrm{Be},{ }^{7} \mathrm{Be}+{ }^{3} \mathrm{H} \leftrightarrow$ $p+{ }^{9} \mathrm{Be}$, which may be relevant in opening new channels for the production of heavier nuclei with $A>7$. The rate for ${ }^{3} \mathrm{He}+{ }^{3} \mathrm{H} \leftrightarrow \gamma+{ }^{6} \mathrm{Li}$ has been also considered and added to the code. This rate was usually neglected since it is assumed that ${ }^{6} \mathrm{Li}$ is mainly produced via ${ }^{4} \mathrm{He}+\mathrm{D} \rightarrow \gamma+{ }^{6} \mathrm{Li}$, whose rate is however poorly known (different low energy non resonant rate estimates disagree for even one or two order of magnitude).

iv) we have analyzed the possible role of some three body processes, as $p+n+n \leftrightarrow n+D$, $n+p+p \leftrightarrow p+D, p+n+n \leftrightarrow \gamma+{ }^{3} H$, and $n+p+p \leftrightarrow \gamma+{ }^{3} H e$. In agreement with standard approach, we checked that these processes can be in fact safely neglected, being suppressed by the high entropy per baryon during BBN.

The benefit of this analysis is of course a higher accuracy in several rates. As a general statement we can say that uncertainties for ${ }^{4} \mathrm{He}$ and $D$ primordial yields are now largely dominated by experimental errors. In principle, if no systematics were present in experimental determinations, a careful analysis of the consistency of the standard BBN scenario would be possible, providing stringent constraints on cosmological parameters $\omega_{b}$ and $N_{\text {eff }}$. Further data, mainly on $\mathrm{D}-\mathrm{D}$ strong reactions and ${ }^{4} \mathrm{He}+{ }^{3} \mathrm{He} \leftrightarrow \gamma+{ }^{7} \mathrm{Be}$, are nevertheless still highly desirable.

As an example of our study we here report some of the main new results on rates 

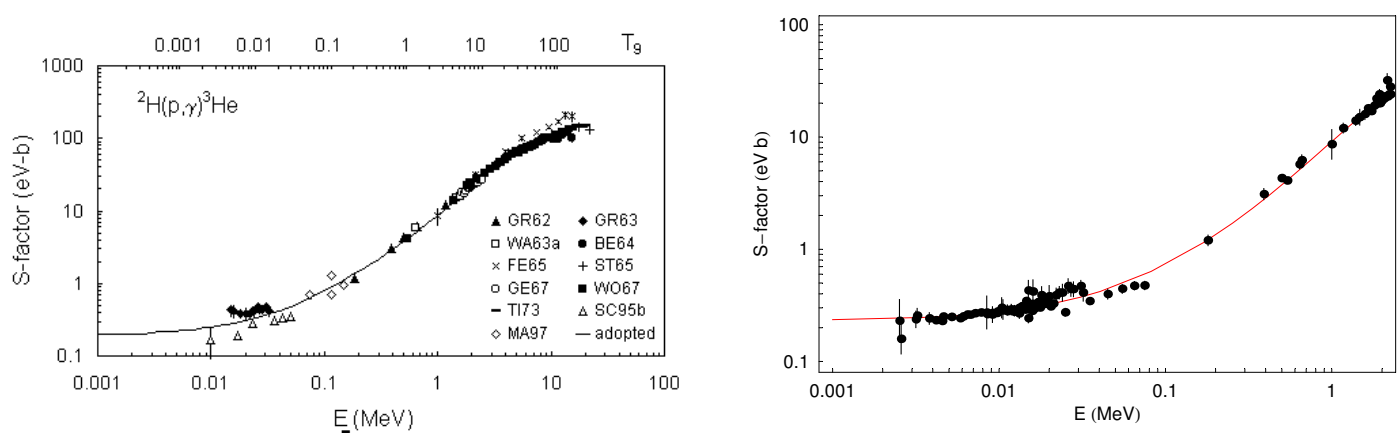

Figure 1: The data and fit for the astrophysical factor of $D+p \rightarrow \gamma+{ }^{3} H e$ from NACRE catalogue (left plot) and with LUNA data added (the fit of $S(E)$ is with a cubic polynomial).

included in the new BBN code. This is by no means a complete overview, which we will present elsewhere [17, but it is enough to illustrate the main aspects of our analysis.

a) $n+p \leftrightarrow \gamma+D$

This reaction is the main process for $D$ production. There are no many experimental results for the corresponding rate in the energy range relevant for $\mathrm{BBN}$ [40]. In the previous versions of the BBN numerical code the corresponding rate estimate 15] was based on the measurement of the thermal capture cross-section, $\sigma_{t h}=$ $(0.3326 \pm 0.0006)$ barn [41], with an overall uncertainty on the rate of $7 \%$.

We have evaluated the rate on the basis of the few low energy data 42 and mainly using the Effective Field Theory. For the low energy range, it is possible to describe the process in terms of an expansion of local operators up to $\mathrm{N}^{4} \mathrm{LO}$, with electromagnetic coupling introduced via gauge principle [43]. The resulting fit of the rate has been then smoothly linked up with the estimate of [15], which is more accurate for $T \geq 0.1 \mathrm{MeV}$. The overall uncertainty is found to be $1.2 \%$ and $7 \%$ for $T \leq 0.1 \mathrm{MeV}$ and $\mathrm{T} \geq 0.1 \mathrm{MeV}$, respectively, which represents a sensible improvement in the relevant region for BBN.

b) $D+p \leftrightarrow \gamma+{ }^{3} H e$

This reaction is the main direct source of ${ }^{3} \mathrm{He}$ together with the strong process $D+D \leftrightarrow n+{ }^{3} H e$. The corresponding rate evaluated in [13] is based on experimental data which are accurate in the energy range $0.1 \div 10 \mathrm{MeV}$, while for lower energies 
there are two different estimates [44, 45]. A fit of the corresponding uncertainty shows a strong temperature dependence, increasing from $5 \%$ at $\mathrm{T} \sim 10 \mathrm{MeV}$ up to $30 \%$ for $T \sim 0.01 \mathrm{MeV}$.

A sensible improvement of accuracy is provided by a reduced incompatibility of the two set of measurements [44, 45], due to the presence of systematics in [44] (see [46]). More recently, the LUNA collaboration has performed a new detailed measurement of this process rate at very low energies [38, close to the solar Gamow peak, $E \sim 6.5 \cdot 10^{-3} \mathrm{MeV}$. We show in Fig. 1 the astrophysical factor as presented in the NACRE catalogue and the fit we have obtained including the new LUNA data. The rate uncertainty is now reduced to at most $3.6 \%$ (statistical error).

c) $D+D \leftrightarrow n+{ }^{3} H e$ and $D+D \leftrightarrow p+{ }^{3} H$

These two reactions are the dominant contribution to, respectively, ${ }^{3} \mathrm{He}$ and ${ }^{3} \mathrm{H}$ production for $T \leq 0.1 \mathrm{MeV}$. Moreover they represent a main source of uncertainty in the theoretical determination of the deuterium final abundance (see i.e. [1] ). We have reanalyzed the data collected in [13, by fitting the (non resonant) astrophysical factor $S(E)$ with a cubic polynomial (in good agreement with the NACRE estimate), but the adopted rate has been fitted with a better accuracy. In our analysis we adopt the NACRE uncertainty. However, an accurate evaluation of the error budget of the data sets, presently in progress [17], seems to indicate that a purely statistical error (below 2\%) can be attained. In any case, even such a large reduction of the rate uncertainties has no relevant consequences on the likelihood analysis, due to the dominance of the experimental errors.

d) ${ }^{3} \mathrm{He}+{ }^{3} \mathrm{H} \leftrightarrow \gamma+{ }^{6} \mathrm{Li}$

This process, modelled with an electric dipole transition [47, is usually neglected in the BBN network, since present estimates suggest that it contributes only for at most $1 \%$ to ${ }^{6} \mathrm{Li}$ production. This nuclide is in fact mainly synthesized via the electric quadrupole transition ${ }^{4} \mathrm{He}+D \leftrightarrow \gamma+{ }^{6} \mathrm{Li}$. Nevertheless, we have decided to include the ${ }^{3} \mathrm{He}+{ }^{3} \mathrm{H} \leftrightarrow \gamma+{ }^{6} \mathrm{Li}$ rate for two reasons. First of all the rate for the process ${ }^{4} \mathrm{He}+\mathrm{D} \leftrightarrow \gamma+{ }^{6} \mathrm{Li}$ is only known for $\mathrm{E}>1 \mathrm{MeV}$ and around the resonance 
at $E=0.711 \mathrm{MeV}$, while at lower energies there are only weak upper limits. On the other hand, the theoretical estimates [13] still differ for order of magnitudes; thus its relative weight in ${ }^{6} \mathrm{Li}$ production may be smaller than expected. Moreover the ${ }^{3} \mathrm{He}+{ }^{3} \mathrm{H} \leftrightarrow \gamma+{ }^{6} \mathrm{Li}$ rate itself is possibly affected by a normalization uncertainty, of the order of a factor 3 (at most) (see Ref. 8 in [13]). Our data fit of the $S$ factor for ${ }^{3} \mathrm{He}+{ }^{3} \mathrm{H} \leftrightarrow \gamma+{ }^{6} \mathrm{Li}$ with a cubic polynomial shows a good agreement with the result of 48 .

We define, as usual, ${ }^{4} \mathrm{He}$ mass fraction, $Y_{p}$, and relative density with respect to hydrogen, $X_{i}\left(i=D,{ }^{3} \mathrm{He},{ }^{6} \mathrm{Li},{ }^{7} \mathrm{Li}\right)$, as

$$
\begin{aligned}
X_{4 H e} \equiv Y_{p} & =4 \frac{n_{4 H e}}{n_{b}}, \\
X_{i} & =\frac{n_{i}}{n_{H}},
\end{aligned}
$$

with $n_{b}$ the baryon density. We do not use ${ }^{7} \mathrm{Li}$ in our analysis to constrain the values of cosmological parameters (see Section 5 for a discussion of this nuclide abundance). To compare the experimental values with theoretical estimates we construct, as customary in literature, a likelihood function $\mathcal{L}$ as follows. If $\Omega$ represents the set of cosmological parameters entering the theoretical model (basically $\omega_{b}$ and $N_{\text {eff }}$ and, in the degenerate BBN scenario, the asymmetry parameter for electron neutrinos, $\xi_{e}$ ), and $R$ the set of all nuclear rates $R_{k}$ involved in the network, the joint $\chi^{2}(\Omega)$ function (i.e. $\left.-2 \log \mathcal{L}\right)$ is defined by

$$
\chi^{2}(\Omega)=\sum_{i j}\left(X_{i}^{t h}(\Omega, R)-X_{i}^{\exp }\right) F_{i j}\left(X_{j}^{t h}(\Omega, R)-X_{j}^{\exp }\right),
$$

where $F$ is the inverse of the error matrix and the sum is over the nuclei considered in the analysis. With $X_{i}^{t h}(\Omega, R)$ and $X_{i}^{\exp }$ we denote the theoretical and experimental estimate for the $i$-nuclide abundance, respectively. In the following we will be interested in studying either the $D$ or the joint $D+{ }^{4} H e$ likelihood function,

$$
\begin{aligned}
-2 \log \left(\mathcal{L}_{D}\right) & =\left(X_{D}^{t h}(\Omega, R)-X_{D}^{e x p}\right)^{2} F_{22} \\
-2 \log \left(\mathcal{L}_{B B N}\right) & =\sum_{i, j=D, 4 H e}\left(X_{i}^{t h}(\Omega, R)-X_{i}^{e x p}\right) F_{i j}\left(X_{j}^{t h}(\Omega, R)-X_{j}^{e x p}\right),
\end{aligned}
$$

For $\mathcal{L}_{D}$

$$
F_{22}^{-1}=\left(\sigma_{22}^{t h}\right)^{2}+\left(\sigma_{22}^{e x p}\right)^{2}
$$


while for the joint likelihood $D+{ }^{4} H e$ the error matrix is given by

$$
F_{i j}^{-1}=\left(\sigma_{i j}^{t h}\right)^{2}+\left(\sigma_{i j}^{e x p}\right)^{2}
$$

i.e. the sum in quadrature of the (diagonal) experimental error matrix $\left(\sigma_{i j}^{e x p}\right)^{2}=\delta_{i j}\left(\sigma_{i}^{e x p}\right)^{2}$ and the theoretical one $\left(\sigma_{i j}^{t h}\right)^{2}(\Omega, R)$. The latter, accounting for the effect on the nuclide abundances of the uncertainties of the several nuclear rates $R_{k}$, can be estimated using the linear propagation method introduced in [11. In a completely general approach, we consider the quantities

$$
\begin{aligned}
\left(\sigma_{i j}^{t h}\right)^{2}(\Omega, R) & \equiv \frac{1}{4} \sum_{k}\left[X_{i}\left(\Omega, R_{k}+\delta R_{k}^{+}\right)-X_{i}\left(\Omega, R_{k}-\delta R_{k}^{-}\right)\right] \\
& \times\left[X_{j}\left(\Omega, R_{k}+\delta R_{k}^{+}\right)-X_{j}\left(\Omega, R_{k}-\delta R_{k}^{-}\right)\right]
\end{aligned}
$$

with $\delta R_{k}^{ \pm}$the (temperature dependent) upper and lower uncertainties on $R_{k}$, respectively. This differs from the approach in [1] which assumes the existence (in principle not necessary) of the linear functionals $\lambda_{i k}=\partial \log X_{i}(\Omega) / \partial \log R_{k}$. This only holds for symmetric and temperature independent relative uncertainties $\delta R_{k} / R_{k}$.

\section{WMAP results and $\omega_{b}$}

Since we are interested in using the CMB data in a joint analysis with the BBN theoretical/experimental ones, the first step in this study has been to produce our own database and likelihood analysis of WMAP measurements. We have used the CMBFAST code [29] and the public software provided by the WMAP collaboration to calculate the likelihood function [30]-33]. The temperature and polarization anisotropies have been evaluated for a set of $\sim 2 \cdot 10^{6}$ models, varying the following parameters in the reported ranges with the corresponding steps:

$$
\begin{array}{cl}
\text { baryon density } & \omega_{b}=\Omega_{b} h^{2}, 0.020 \div 0.029, \Delta \omega_{b}=0.0015 \\
\text { cold dark matter density } & \omega_{c}=\Omega_{c} h^{2}, 0.027 \div 0.236, \Delta \omega_{c}=0.011 \\
\text { Hubble parameter } & h, 0.6 \div 0.8, \Delta h=0.017 \\
\text { optical depth } & \tau, 0 \div 0.4, \Delta \tau=0.05 \\
\text { scalar tilt } & n_{s}, 0.8 \div 1.2, \Delta n_{s}=0.036 \\
\text { relativistic particle density } & N_{\text {eff }}, 0 \div 9, \Delta N_{\text {eff }}=1 .
\end{array}
$$




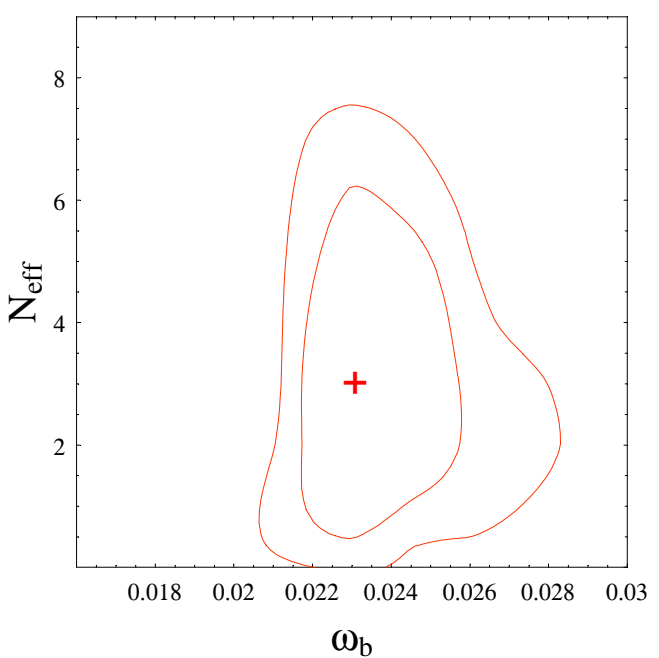

Figure 2: The 68 and 95\% C.L. likelihood contours from WMAP data in the $N_{e f f}-\omega_{b}$ plane.

We only consider spatially flat models, as suggested by the position of the first acoustic peak, now determined by WMAP with a rather high accuracy, $l_{p}=220.1 \pm 0.8$ [49]. We have also let as a free parameter the overall normalization, which is eventually fixed by the likelihood analysis. In Table 1 we report the best fit values and 95\% C.L. ranges obtained after marginalization over remaining parameters. Notice that $h$ does not appear in this Table since we consider its range as a prior.

By using as fitting parameters $\omega_{b}, \omega_{c}, h, \tau$ and $n_{s}$ and fixing $N_{\text {eff }}=3.04$ one gets at the best fit $\chi^{2}=1431$ for 1342 d.o.f. . The introduction of $N_{\text {eff }}$ as a new free parameter lowers the value of $\chi^{2}$ to 1430 for 1341 d.o.f., not significantly improving the goodness of fit. This is in perfect agreement with the results of [24].

The results are shown in Fig. 2,3, After marginalization we get $\omega_{b}=0.023_{-0.001}^{+0.002}$ and $N_{e f f}=2.6_{-1.5}^{+2.0}$, at $68 \%$ C.L., in good agreement with similar results obtained in the recent

\begin{tabular}{|c|c|c|c|c|c|}
\hline \hline & $n_{s}$ & $\omega_{c}$ & $\omega_{b}$ & $\tau$ & $N_{\text {eff }}$ \\
\hline \hline marginalized $(2 \sigma)$ & $0.98_{-0.09}^{+0.11}$ & $0.11_{-0.06}^{+0.09}$ & $0.023_{-0.002}^{+0.004}$ & $0.12_{-0.09}^{+0.22}$ & $2.6_{-2.4}^{+3.9}$ \\
\hline best fit & 0.982 & 0.115 & 0.023 & 0.15 & 3.0 \\
\hline
\end{tabular}

Table 1: The best fit values and the $(2 \sigma)$ bounds for marginalized distributions are here reported for the fit analysis of WMAP data. 

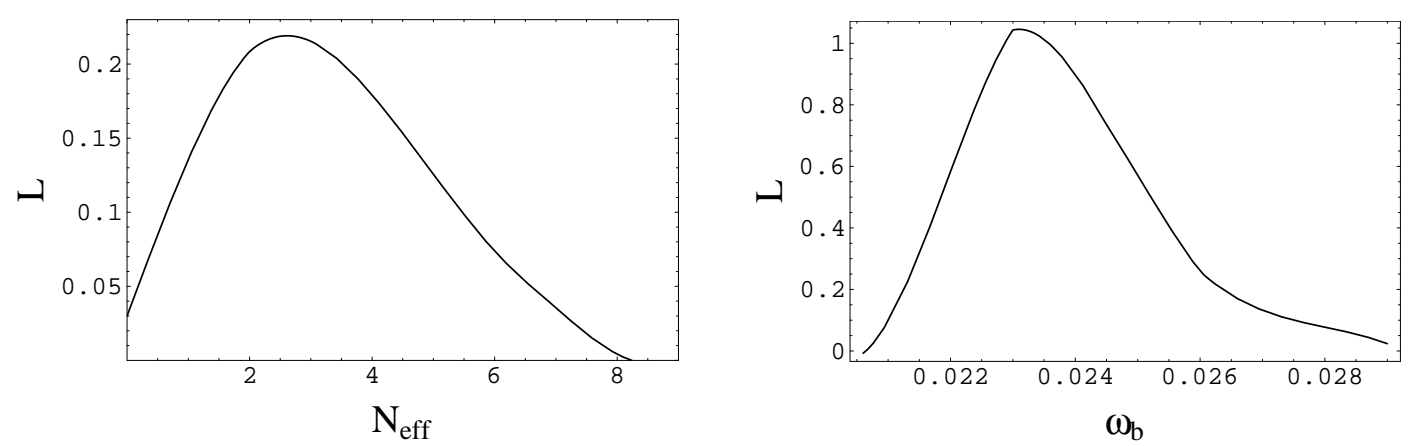

Figure 3: The marginalized likelihoods versus $N_{\text {eff }}$ and $\omega_{b}$.

literature [24]-26]. Compare, for example, our Fig. 3 for $N_{\text {eff }}$ with the red curve in Fig. 1 of Ref. 24]. We note that the range obtained in [28] is instead slightly larger.

For completeness we report in Fig. 4 bidimensional likelihood contours in all planes involving at least one of the parameters $\omega_{b}, N_{\text {eff }}$. From this figure we see how CMB data alone cannot break the degeneracies $N_{e f f}-h$ and $N_{e f f}-\omega_{c}$, which are the main sources of uncertainty in the determination of $N_{\text {eff }}$. Removing these degeneracies requires other data sets than CMB. Notice that the ripples in the contours are an artefact of the finite size in the parameter lattice.

\section{$4 \mathrm{CMB}+\mathrm{BBN}($ Deuterium) analysis}

Deuterium number fraction, $X_{D}$, is critically depending on the baryon content of the universe. It is therefore worth starting our analysis of the consistency of the CMB + BBN standard scenario by discussing how WMAP determination of $\omega_{b}$ fits with the experimental determination of $X_{D}$.

The estimate of $X_{D}$ is affected by a theoretical uncertainty which is dominated by a small number of nuclear rate uncertainties, mainly the processes which produces either ${ }^{3} \mathrm{H}$ or ${ }^{3} \mathrm{He}$ out of Deuterium. Varying all the rates $R_{k}$ which enter the network with $1 \sigma$ errors $\delta R_{k}^{ \pm}$(see Section 2), we get from our code, for $\omega_{b}=0.023, \sigma_{22}^{t h}=0.15 \cdot 10^{-5}$. In Table 2 we also show the relative contribution of the main processes to this result, in percentage.

Our previous estimate for the same parameter was $\sigma_{22}^{t h}=0.21 \cdot 10^{-5}[9]$. The main source of this improvement is due both to the more precise data which are now available 

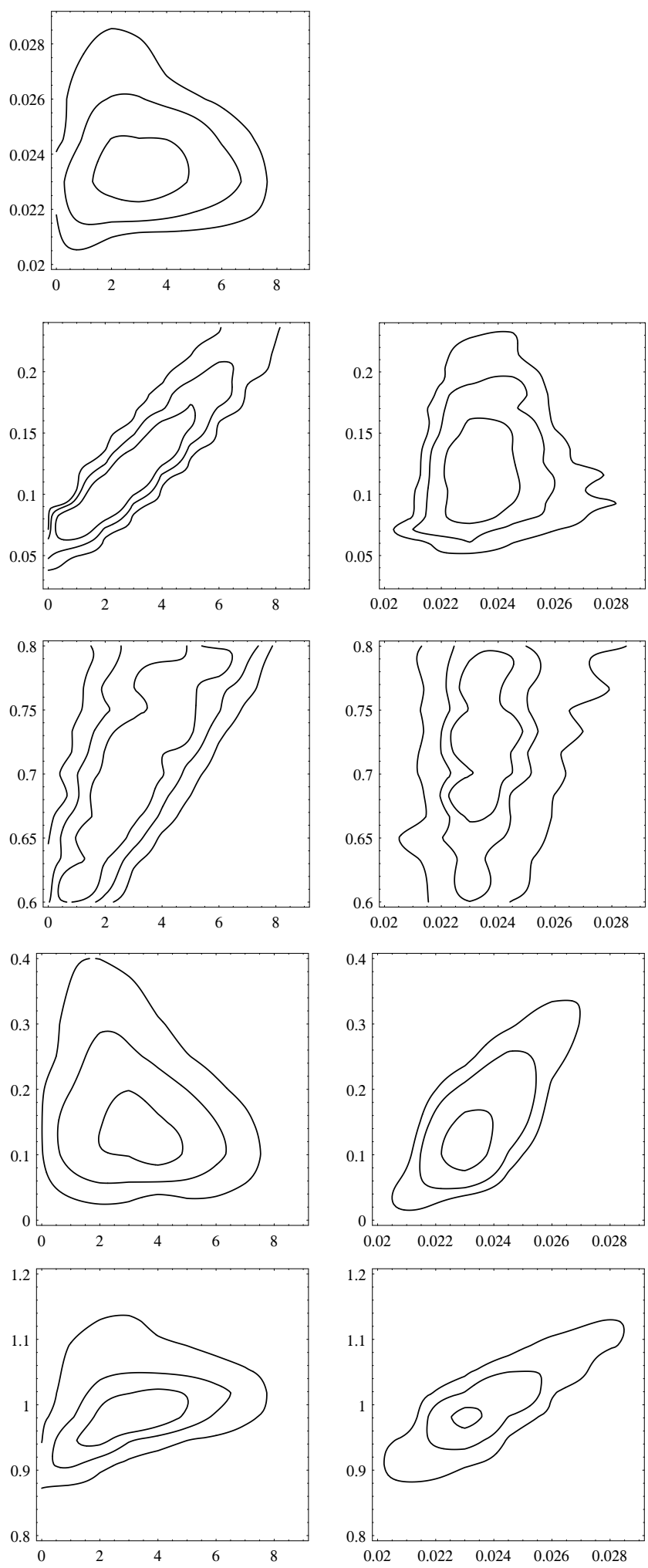

Figure 4: Arbitrary levels of the bidimensional marginalized likelihood contours: $x$-axis in first (second) column corresponds to $N_{\text {eff }}\left(\omega_{b}\right)$, from top to bottom $y$-axis corresponds to $\omega_{b}, \omega_{c}, h, \tau$ and $n_{s}$, respectively. 


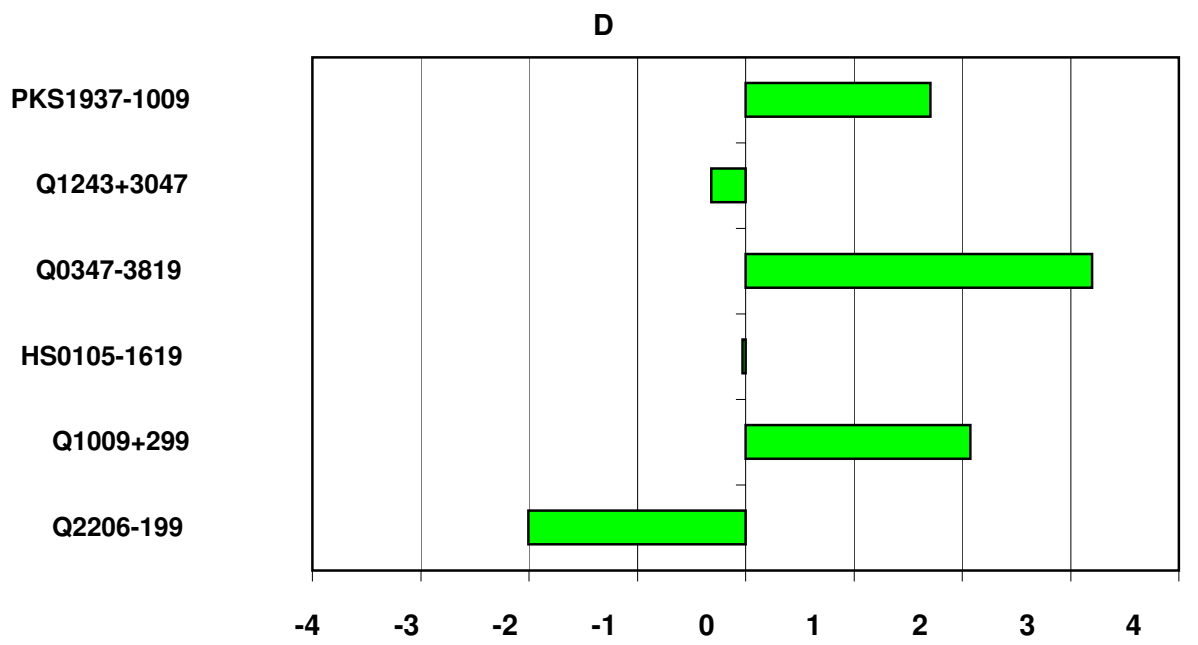

Figure 5: The pulls (see text) of QSO D measurements with respect to the theoretical prediction for $N_{e f f}=3.01$ and $\omega_{b}=0.023$, in units of $\left(\left(\sigma_{22}^{\text {th }}\right)^{2}+\left(\sigma_{2}^{e x p}\right)^{2}\right)^{1 / 2}$.

on $D+p \leftrightarrow \gamma+{ }^{3} \mathrm{He}$ and the reanalysis of the $D-D$ rates. Considering $\sigma_{22}^{t h}$ and the allowed range for $\omega_{b}$ obtained in Section 3, $0.022 \leq \omega_{b} \leq 0.025$, we get, for the standard value $N_{\text {eff }}=3.01, X_{D}=\left(2.55_{-0.34}^{+0.22}\right) \cdot 10^{-5}$. This theoretical prediction nicely fits, within the errors, with the most recent experimental results of [4, $X_{D}=\left(2.78_{-0.38}^{+0.44}\right) \cdot 10^{-5}$. The latter is the average of several measurements of $D I / H I$ column ratio in different QSO absorption systems at high red-shift.

In Fig. [5] we report the deviations of the experimental values of $X_{D}$ with respect to the theoretical prediction of standard BBN, in unit of the combined error due to $\sigma_{22}^{\text {th }}$ and $\sigma_{2}^{\text {exp }}$ summed in quadrature (pulls). In spite of the fact that unidentified effects may be still affecting some of the data, it is nevertheless interesting to note that, as far as Deuterium is concerned, the standard BBN scenario with three neutrinos and WMAP result for $\omega_{b}$ is quite consistent.

\begin{tabular}{|c|c|}
\hline \hline rate $R_{k}$ & $\delta\left(\sigma_{22 k}^{t h}\right)^{2} /\left(\sigma_{22}^{t h}\right)^{2}(\%)$ \\
\hline \hline$D+D \leftrightarrow n+{ }^{3} H e$ & 73 \\
$D+D \leftrightarrow p+{ }^{3} H$ & 23 \\
$D+p \leftrightarrow \gamma+{ }^{3} H e$ & 3 \\
$p+n \leftrightarrow \gamma+D$ & 0.6 \\
\hline \hline
\end{tabular}

Table 2: The main contributions to $\left(\sigma_{22}^{t h}\right)^{2}$, in percentage, for $N_{\text {eff }}=3.01$ and $\omega_{b}=0.023$. 

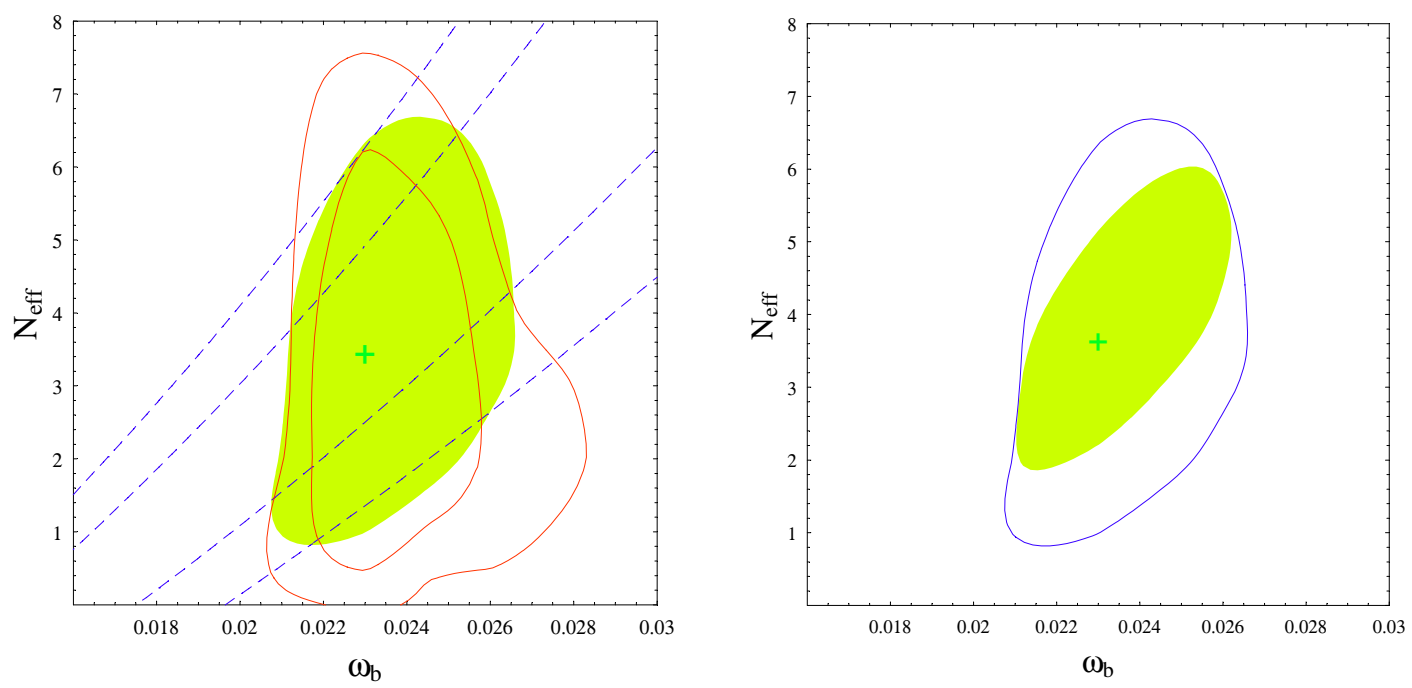

Figure 6: Left plot: the 68 and 95\% C.L. contours in the $\omega_{b}-N_{\text {eff }}$ plane for WMAP (solid lines). The dashed lines are two arbitrary levels of the BBN Deuterium likelihood. The joint $95 \%$ C.L. $C M B+B B N$ region is shown as the filled area. Right plot: the joint $95 \%$ C.L. contour of the left plot (solid line) is compared to the same contour with a total error on $X_{D}$ improved by a factor 2 (filled area).

To analyze this point more quantitatively we have performed a likelihood study using the WMAP data and BBN Deuterium abundance only. The likelihood function for $D$ has been constructed as described in Section 2. The $N_{\text {eff }}$ and $\omega_{b}$ dependence of $\sigma_{22}^{\text {th }}$ has been fully taken into account. The result is shown in Fig. 6 (left plot), from which we see the good agreement of a standard BBN with three active neutrinos with the WMAP determination of $\omega_{b}$. The product likelihood $\mathrm{CMB}+\mathrm{BBN}$ is peaked at the values $\omega_{b}=$ 0.023 and $N_{e f f}=3.4$. Likelihood marginalization gives, at $95 \%$ C.L., $\omega_{b}=0.023_{-0.002}^{+0.003}$ and $N_{\text {eff }}=3.6_{-2.3}^{+2.5}$, which give at $1 \sigma X_{D}=\left(2.74_{-0.51}^{+0.46}\right) \cdot 10^{-5}, Y_{p}=0.256_{-0.015}^{+0.014}$ and $X_{7 L i}=\left(4.7_{-1.3}^{+1.5}\right) \cdot 10^{-10}$. As expected, $N_{e f f}$ is not severely constrained either by $X_{D}$ or from CMB: with present uncertainties on Deuterium, the 95\% C.L. interval corresponds to $1.3 \leq N_{e f f} \leq 6.1$. Previous values, when comparisons are possible, are in nice agreement with the results reported in [24, 26]. A more stringent bound on $N_{\text {eff }}$ could be provided by ${ }^{4} \mathrm{He}$ mass fraction. We will discuss this in more details in the next Section. It is however interesting to see whether the value of $N_{\text {eff }}$ could be more precisely fixed by Deuterium+WMAP if new and more precise measurements from QSO were available. We show in Fig. 6 (right plot) the results of a simulation with an error reduced by a factor two, $\sqrt{\left(\sigma_{22}^{\text {th }}\right)^{2}+\left(\sigma_{2}^{\exp }\right)^{2}} \simeq 0.2 \cdot 10^{-5}$. In this case, repeating the same analysis, we obtain 
$2.3 \leq N_{\text {eff }} \leq 5.7$, still a broad range, but not much wider than what is obtained using a customary BBN likelihood analysis of both $\mathrm{D}$ and ${ }^{4} \mathrm{He}$.

\section{$5 \quad \mathrm{BBN}\left(\right.$ Deuterium $\left.+{ }^{4} H e\right)$ analysis}

The theoretical estimates for ${ }^{4} \mathrm{He}$ mass fraction $Y_{p}$ and ${ }^{7} \mathrm{Li}$ number density $X_{7 L i}$ show a slight disagreement with the corresponding experimental results. For $N_{\text {eff }}=3.01$ and using the WMAP result for $\omega_{b}$ we get $Y_{p}=0.2483_{-0.0005}^{+0.0008}$ and $X_{7 L i}=\left(4.9_{-1.2}^{+1.4}\right) \cdot 10^{-10}$. The source of uncertainties for these two nuclei is as follows. For $Y_{p}$ the propagated uncertainty due to nuclear rates, $\sigma_{44}^{t h}=0.0003$, is of the same order of magnitude of the one due to the variation of $\omega_{b}$. Notice how the smaller error on neutron lifetime, $\Delta \tau_{n}=0.8 s$, has strongly reduced the effects on $\left(\sigma_{44}^{t h}\right)^{2}$ of weak $n \leftrightarrow p$ processes. Concerning the $X_{7 L i}$ uncertainty, this is mainly due to the ${ }^{4} \mathrm{He}+{ }^{3} \mathrm{He} \leftrightarrow \gamma+{ }^{7} \mathrm{Be}$ rate, with $\omega_{b}$ contributing for $\sim 50 \%$ to the total error on $X_{7 L i}$. We show in Table 3 the relative contribution of the most important rates to $\left(\sigma_{44}^{t h}\right)^{2}$ and $\left(\sigma_{77}^{t h}\right)^{2}$.

The status of the primordial abundance measurements for ${ }^{4} \mathrm{He}$ and ${ }^{7} \mathrm{Li}$ is still quite involved. There are different determinations of $Y_{p}, Y_{p}=0.234 \pm 0.003$ [50] (low $Y_{p}$ ), $Y_{p}=0.244 \pm 0.002$ [51] (high $Y_{p}$ ), and $Y_{p}=0.2421 \pm 0.0021$ [52]. The latter is the result of a recent analysis where the authors performed an accurate study of systematics. If the statistical error were not underestimated, these values would be only marginally compatible. Waiting for new experimental data, we adopt in our analysis a conservative point of view by using

$$
Y_{p}=0.239 \pm 0.008
$$

\begin{tabular}{|c|c|c|c|}
\hline \hline rate $R_{k}$ & $\left(\delta \sigma_{44 k}^{t h} / \sigma_{44}^{t h}\right)^{2}(\%)$ & rate $R_{k}$ & $\left(\delta \sigma_{77 k}^{\text {th }} / \sigma_{77}^{\text {th }}\right)^{2}(\%)$ \\
\hline \hline$n \leftrightarrow p$ & 29 & ${ }^{4} \mathrm{He}+{ }^{3} \mathrm{He} \leftrightarrow \gamma+{ }^{7} \mathrm{Be}$ & 68 \\
$D+D \leftrightarrow n+{ }^{3} \mathrm{He}$ & 29 & ${ }^{3} \mathrm{He}+\mathrm{D} \leftrightarrow p+{ }^{4} \mathrm{He}$ & 9 \\
$D+D \leftrightarrow p+{ }^{3} H$ & 26 & $D+D \leftrightarrow n+{ }^{3} \mathrm{He}$ & 8.5 \\
$p+n \leftrightarrow \gamma+D$ & 4 & ${ }^{7} \mathrm{Be}+n \leftrightarrow{ }^{4} \mathrm{He}+{ }^{4} \mathrm{He}$ & 7 \\
\hline \hline
\end{tabular}

Table 3: The main contributions to $\left(\sigma_{44}^{t h}\right)^{2}$ and $\left(\sigma_{77}^{t h}\right)^{2}$, in percentage, for $N_{\text {eff }}=3.01$ and $\omega_{b}=0.023 . n \leftrightarrow p$ denotes the weak processes. 


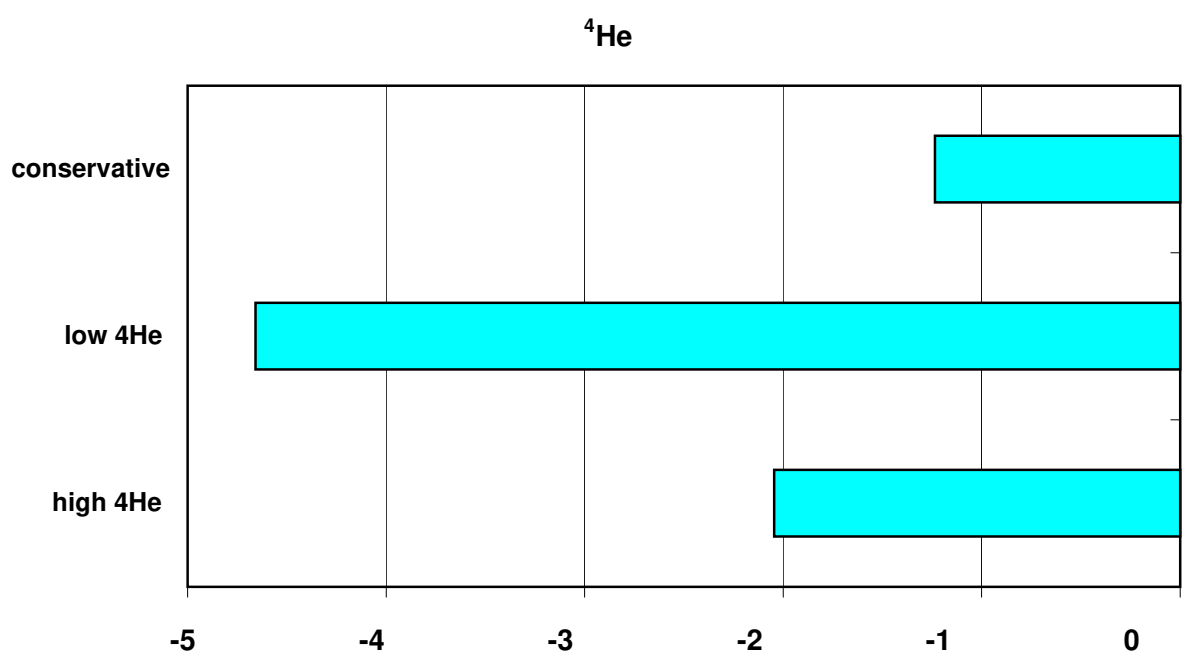

Figure 7: The pulls (see text) of $Y_{p}$ measurements with respect to the theoretical prediction for $N_{\text {eff }}=3.01$ and $\omega_{b}=0.023$, in units of $\left(\left(\sigma_{44}^{t h}\right)^{2}+\left(\sigma_{4}^{e x p}\right)^{2}\right)^{1 / 2}$.

As it is evident from Fig. 7] where we report the pulls with respects to expected theoretical $Y_{p}$ in unit of the combined error $\left(\left(\sigma_{44}^{t h}\right)^{2}+\left(\sigma_{4}^{e x p}\right)^{2}\right)^{1 / 2}$, even the higher value of $Y_{p}$ [51] appears in moderate disagreement ( $2 \sigma$ effect) with standard BBN. If we let $N_{\text {eff }}$ to be a free parameter it is therefore not a surprise that $Y_{p}$ favors a slightly lower value for $N_{e f f}$. In fact, a way to decrease $Y_{p}$ is to delay the freezing out of weak rates converting neutron and protons, and this can be achieved by reducing the number of relativistic species contributing to the Hubble parameter. In our opinion much effort should be devoted to have a more clear understanding of the experimental results or to undertake a new measurement campaign for ${ }^{4} \mathrm{He}$. On the theoretical side, in fact, we may say that the value of $Y_{p}$ is quite robust, and affected by a very small total error. New data would eventually tell us whether the standard BBN is fully consistent, or more exotic scenarios should be invoked. We discuss one of these, a degenerate neutrino distribution, in next Section.

Concerning ${ }^{7} \mathrm{Li}$ experimental measurements [53]-[57], the primordial origin of the Spite plateau has been recently questioned. In particular the authors of [54 found evidence for a dependence of $X_{7 L i}$ on metallicity. In any case, there are some indication for a depletion mechanism of ${ }^{7} \mathrm{Li}$ observed in POP II metal poor stars. However, as can be seen in Fig. 8 , the discrepancy between the most recent observations of [55]- [57] and our theoretical value 


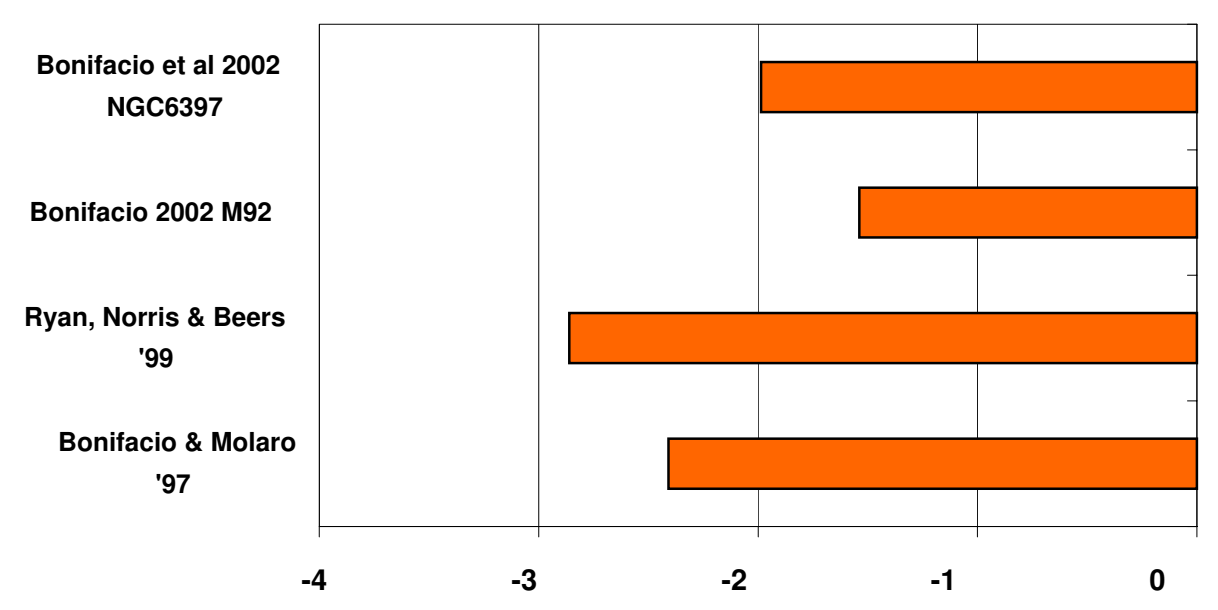

Figure 8: The pulls (see text) of $X_{7 L i}$ measurements with respect to the theoretical prediction for $N_{e f f}=3.01$ and $\omega_{b}=0.023$, in units of $\left(\left(\sigma_{77}^{t h}\right)^{2}+\left(\sigma_{7}^{e x p}\right)^{2}\right)^{1 / 2}$.

is now reduced to a less than $3 \sigma$ effect. The average over the different observed values for $X_{7 L i}$ [53]-[57], which are mutually compatible, gives $X_{7 L i}=(1.70 \pm 0.17) \times 10^{-10}$.

As in previous studies we do not use $X_{7 L i}$ in our BBN likelihood analysis, waiting for a more clear understanding of a possible depletion mechanism. Moreover, further efforts should be also devoted in trying to reduce the large theoretical uncertainty which is still affecting $X_{7 L i}$, mainly by lowering the uncertainty on the leading reaction ${ }^{4} \mathrm{He}+{ }^{3} \mathrm{He} \leftrightarrow$ $\gamma+{ }^{7} \mathrm{Be}$, presently of the order of $18 \%$. As we mentioned, this produces a large fraction of the overall $20 \%$ error on $X_{7 L i}$.

Using the $X_{D}$ result of [4] and Eq. (5.1) for $Y_{p}$, we consider the likelihood function as defined in (2.4), as function of $N_{\text {eff }}$ and $\omega_{b}$. Our results for 68 and 95\% C.L. contours are shown in Fig. 9, where for comparison we also report the 95\% C.L. contour of the $C M B+D$ analysis. The effect of ${ }^{4} H e$ has been to shift both $N_{\text {eff }}$ and $\omega_{b}$ towards smaller values. In particular, after marginalization, we get at $2 \sigma, \omega_{b}=0.021_{-0.004}^{+0.005}$ and $N_{\text {eff }}=2.5_{-0.9}^{+1.1}$. Notice how, with present determination of $Y_{p}$ and $X_{D}$, the range obtained for $\omega_{b}$ is rather broad compared with WMAP result.

We close this section with the theoretical estimate on ${ }^{3} \mathrm{He}$ and ${ }^{6} \mathrm{Li}$ number fractions. For $\omega_{b}=0.021$ and $N_{e f f}=2.5$ we get $X_{3 H e}=\left(1.02_{-0.11}^{+0.10}\right) \cdot 10^{-5}$, which saturates the recent estimate reported in [58] for the upper limit of the ${ }^{3} H e$ to $H$ number density 


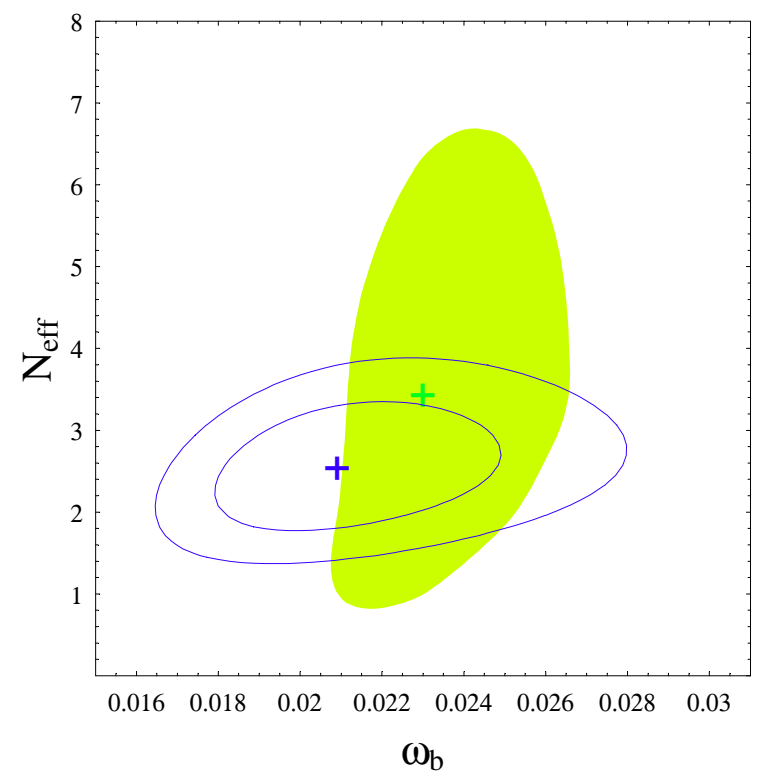

Figure 9: The 68 and 95\% C.L. contours for the $D+{ }^{4} \mathrm{He}$ likelihood function in the $\omega_{b}-N_{\text {eff }}$ plane. We also show the result of the $C M B+D$ analysis of Fig. [6 (left plot).

from observations of $H I I$ regions and planetary nebulae, $X_{3 H e}=(1.1 \pm 0.2) \cdot 10^{-5}$. The ${ }^{6} \mathrm{Li}$ primordial density is predicted to be quite small, $X_{6 L i}=(1.2 \pm 1.7) \cdot 10^{-14}$, which results in a ratio ${ }^{6} \mathrm{Li} /{ }^{7} \mathrm{Li}=(2.8 \pm 4.0) \cdot 10^{-5}$, much smaller than the experimental value ${ }^{6} \mathrm{Li} /{ }^{7} \mathrm{Li}=0.05$, measured in two stars in the galactic halo (see, for example, [59] and refs. therein). Very close result are obtained for the other analysis considered in the previous Section. We recall however that the theoretical estimate is still suffering the large uncertainty affecting the rate ${ }^{4} \mathrm{He}+D \leftrightarrow \gamma+{ }^{6} \mathrm{Li}$, which is supposed to give the main production channel during BBN (see our discussion in Section 2). Actually any ${ }^{6} \mathrm{Li}$ detection has important consequences in constraining the destruction rate of ${ }^{7} \mathrm{Li}$ in POP II stars, as well as exotic processes to $D$ synthesis, as for example photodissociation or spallation of ${ }^{4} H e$ [60]. A summary of the ranges for $\omega_{b}$ and $N_{\text {eff }}$ and of nuclei yields is reported in Table 4.

\section{Bounds on neutrino chemical potentials. Still room for extra relativistic species}

The cosmological effects of non zero neutrino antineutrino asymmetries have received 


\begin{tabular}{|c|c|c|c|}
\hline \hline & $\begin{array}{c}C M B+B B N(D) \\
\text { at } N_{\text {eff }}=3.01\end{array}$ & $C M B+B B N(D)$ & $B B N\left(D+{ }^{4} H e\right)$ \\
\hline \hline$\omega_{b}(2 \sigma)$ & $0.023_{-0.002}^{+0.003}$ & $0.023_{-0.002}^{+0.003}$ & $0.021_{-0.004}^{+0.005}$ \\
\hline$N_{\text {eff }}(2 \sigma)$ & 3.01 & $3.6_{-2.3}^{+2.5}$ & $2.5_{-0.9}^{+1.1}$ \\
\hline $\begin{array}{c}X_{D} \cdot 10^{5}(1 \sigma) \\
\left(2.78_{-0.38}^{+0.44}\right.\end{array}$ & $2.55_{-0.34}^{+0.22}$ & $2.74_{-0.51}^{+0.46}$ & $2.75_{-0.57}^{+0.53}$ \\
\hline $\begin{array}{c}Y_{p}(1 \sigma) \\
(0.239 \pm 0.008)\end{array}$ & $0.2483_{-0.0005}^{+0.0008}$ & $0.256_{-0.015}^{+0.014}$ & $0.240 \pm 0.008$ \\
\hline $\begin{array}{c}X_{7 L i} \cdot 10^{10}(1 \sigma) \\
(1.70 \pm 0.17)\end{array}$ & $4.9_{-1.2}^{+1.4}$ & $4.7_{-1.3}^{+1.5}$ & $4.3_{-1.3}^{+1.6}$ \\
\hline $\begin{array}{c}X_{3 H e} \cdot 10^{5}(1 \sigma) \\
(1.1 \pm 0.2)\end{array}$ & $0.99_{-0.08}^{+0.07}$ & $1.01_{-0.10}^{+0.09}$ & $1.02_{-0.11}^{+0.10}$ \\
\hline $\begin{array}{c}X_{6 L i} / X_{7 L i}(1 \sigma) \\
(0.05)\end{array}$ & $(2.4 \pm 3.4) \cdot 10^{-5}$ & $(2.8 \pm 3.7) \cdot 10^{-5}$ & $(2.8 \pm 4.0) \cdot 10^{-5}$ \\
\hline
\end{tabular}

Table 4: A summary of the results obtained in the three different SBBN likelihood analysis discussed in this work. For nuclear abundances we report in parenthesis the experimental value or the best estimate currently available: actually, only in some cases a direct comparison is possible, as explained in the text. The larger uncertainties affecting $Y_{p}$ in the third and fourth columns are due to the variation of $N_{\text {eff }}$ in the $1-\sigma$ range. 
a renewed attention in the last few years [23, 61]-65]. The bounds from BBN and CMB data on the asymmetry parameters $\xi_{\alpha}$ for the three active neutrinos, $\alpha=e, \mu, \tau$, are more stringent by considering the effect of oscillations in primordial universe. In fact, in [35] it was shown that for the large mixing angle solution to solar neutrino problem, presently favored by data, oscillations tend to homogenize the $\xi_{\alpha}$, which are therefore all bounded by the more stringent constraint on $\xi_{e}$. Neutrino chemical potentials affect BBN via their contribution to energy density,

$$
\rho_{\nu}=\frac{7}{8}\left(\frac{T_{\nu}}{T_{\gamma}}\right)^{4} \rho_{\gamma}\left\{3.01+\sum_{\alpha}\left[\frac{30}{7}\left(\frac{\xi_{\alpha}}{\pi}\right)^{2}+\frac{15}{7}\left(\frac{\xi_{\alpha}}{\pi}\right)^{4}\right]\right\},
$$

and so to Hubble parameter. In particular $\xi_{\mu, \tau}$ only enters in changing the value of $N_{\text {eff }}$. On the other hand $\xi_{e}$ also affects the $n / p$ chemical equilibrium at freeze out, changing the neutron to proton ratio as $\exp \left(-\xi_{e}\right)$. A positive $\xi_{e}$ (more neutrinos than antineutrinos) favors $n \rightarrow p$ reactions with respect to inverse processes, and this eventually translates into a smaller value for $Y_{p}$.

In this section, using the WMAP result on $\omega_{b}$ as a prior, we use our BBN code to update our previous analysis [2] of degenerate BBN (DBBN), with two main purposes:

i) since, as we stressed in the previous Section, the $Y_{p}$ experimental value is slightly lower than what expected in standard BBN, we analyze how the agreement improves allowing for non zero $\xi_{\alpha}$, but fixing the number of relativistic species, besides photons, to the three active light neutrinos.

ii) in the general case we determine the upper bounds in DBBN on $N_{\text {eff }}$ and $\xi_{\alpha}$.

We construct the BBN likelihood function $\mathcal{L}_{B B N}\left(N_{e f f}, \omega_{b}, \xi_{e}\right)$ defined in Eq. (2.4), with the extra dependence on the parameter $\xi_{e}$. The use of $Y_{p}$ may seem inconsistent with our discussion of the previous Section. For standard BBN in fact we stressed that the experimental value of $Y_{p}$ shows a slight disagreement with the theoretical estimate, possibly a sign of unaccounted systematic effects. However the introduction of a (positive) $\xi_{e}$ in the DBBN case leads to a smaller theoretical value for $Y_{p}$ even for $N_{\text {eff }} \sim 3$, so in this case one may interpret the experimental findings as an indication of a neutrinoantineutrino asymmetry. 

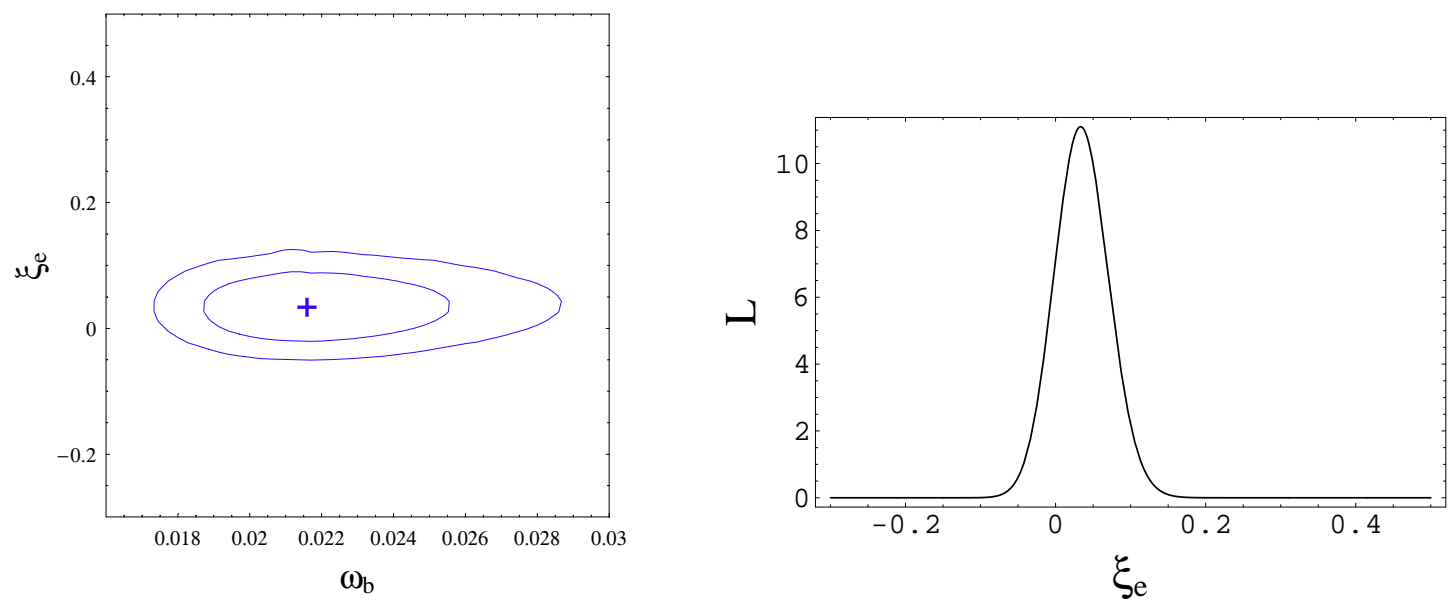

Figure 10: The 68 and $95 \%$ contours for the $\mathrm{D}+{ }^{4} \mathrm{He}$ degenerate likelihood function in the $\omega_{b}-\xi_{e}$ plane in the scenario with only three active neutrinos and without prior on $\omega_{b}$ (left plot). Right plot shows the marginalized likelihood versus $\xi_{e}$ when a prior on $\omega_{b}$ is imposed, $\omega_{b}=0.023 \pm 0.002$.

In the minimal scenario we assume only three active neutrinos contributing to $N_{e f f}$. Since $\xi_{\mu, \tau}$ are enforced by oscillations to be of the same order of magnitude of $\xi_{e}$, we can write down a constraint for $N_{\text {eff }}$,

$$
N_{e f f}=3.01+3\left[\frac{30}{7}\left(\frac{\xi_{e}}{\pi}\right)^{2}+\frac{15}{7}\left(\frac{\xi_{e}}{\pi}\right)^{4}\right]=\bar{N}_{e f f}\left(\xi_{e}\right)
$$

In the second scenario we parameterize possible extra light degrees of freedom with $\Delta N_{\text {eff }}$, defined by

$$
N_{e f f}=\bar{N}_{e f f}\left(\xi_{e}\right)+\Delta N_{e f f}
$$

In both these cases we adopt the experimental values/errors for $Y_{p}$ and $X_{D}$ reported in Section 4 and 5 .

We show in Fig.10 (left plot) the 68 and 95\% C.L. contours for the first scenario. When imposing the WMAP prior with a conservative error $\left(\omega_{b}=0.023 \pm 0.002\right)$ we obtain the marginalized likelihood for $\xi_{e}$ reported in the right plot of Fig. 10, giving $\xi_{e}=0.03_{-0.06}^{+0.08}$ $(2 \sigma)$. Correspondingly $X_{D}=2.51 \cdot 10^{-5}, Y_{p}=0.2414$.

Fig. 11] summarizes our findings in the second scenario. At 95\% C.L., we get $N_{e f f}=$ $3.9_{-3.4}^{+4.8}$ and $\xi_{e}=0.13_{-0.26}^{+0.18}$, again using the WMAP prior on $\omega_{b}$. We notice that our limits on $N_{\text {eff }}$ are broader than what found in [34], since we use only the $\omega_{b}$ prior from CMB in the analysis. This results in a more conservative bound, independent on the assumption 


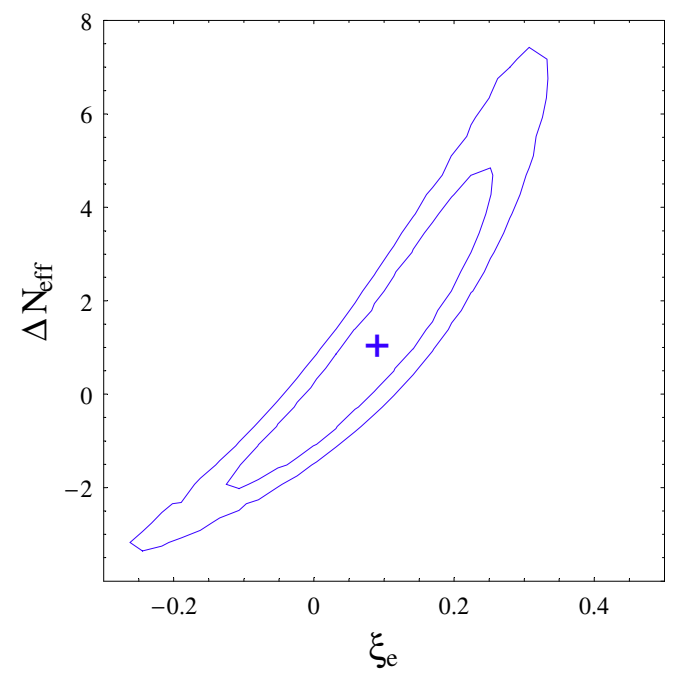

Figure 11: The 68 and $95 \%$ C.L. contours in the $\xi_{e}-\Delta N_{\text {eff }}$ plane for the degenerate BBN likelihood function.

that $N_{\text {eff }}$ is the same at both the CMB and BBN epochs. Our upper limit on $N_{\text {eff }}$, being sensibly larger than 3, should be understood as a direct bound to possible extra light particles. Notice that in the DBBN scenario a fourth sterile neutrino, as for example required to interpret LSND evidence for $\bar{\nu}_{\mu} \leftrightarrow \bar{\nu}_{e}$ oscillation, is not yet ruled out. See also 26] for a neutrino mass analysis of this issue.

\section{Conclusions}

In this paper we have discussed the present status of Primordial Nucleosynthesis after the recent measurements of CMB anisotropies by the WMAP experiment, which represents a new relevant step towards a better understanding of the evolution of the universe, and in particular gives a very precise determination of the baryon density parameter $\omega_{b}$. The question of whether the standard picture of BBN is a satisfactory scenario for production of light nuclei requires an increased precision in both its theoretical and experimental aspects.

As discussed in the paper, it seems to us that some results in this direction have already been achieved. The accuracy of theoretical estimates has been improved by refining the estimate of $n / p$ ratio at decoupling. Most importantly, a complete reanalysis of all nuclear rates which enter the $\mathrm{BBN}$ network has been made, using the NACRE compilation, as 
well as all most recent available results. This analysis has been used to implement a new BBN numerical code. Particular attention has been devoted to the rates which mainly contribute to evolution of observed nuclei abundances. Moreover several processes which were not considered in previous analysis have been added. The main outcome of this study is a reduced theoretical uncertainty of the BBN model, which is presently well below the corresponding experimental errors, at least for $\mathrm{D}$ and ${ }^{4} \mathrm{He}$. The ${ }^{7} \mathrm{Li}$ number fraction is still affected by a $20 \%$ uncertainty, mainly due to the effect of ${ }^{4} \mathrm{He}+{ }^{3} \mathrm{He} \leftrightarrow \gamma+{ }^{7} \mathrm{Be}$ rate. More accurate measurements of this rate are therefore strongly desirable.

From the experimental point of view, Deuterium determinations show a very good agreement with the corresponding theoretical expectation, and are fully compatible with the value of $\omega_{b}$ suggested by WMAP data. This is quite remarkable, since $D$ is strongly dependent on the baryon content of the universe. On the other hand we do think that new measurements of ${ }^{4} \mathrm{He}$ and ${ }^{7} \mathrm{Li}$ should be performed, along with a clear understanding of possible depletion mechanisms of primordial $X_{7 L i}$. It should be mentioned however that the most recent results for ${ }^{7} \mathrm{Li}$, compared with our theoretical prediction, reduce the disagreement for $X_{7 L i}$ at less than $3 \sigma$ level. The ${ }^{4} H e$ mass fraction still has two distinct determinations which are mutually incompatible. Even adopting a conservative value, as we did in this paper, still the amount of ${ }^{4} \mathrm{He}$ experimentally detected seems slightly lower than expected for a standard BBN scenario. This may be seen either as due to possible systematics in the measurements and/or extrapolation technique for $Y_{p}$, or rather as the effect of exotic physics in the BBN scenario, as for example neutrino degeneracy. For an alternative method, still affected by large uncertainty, to infer $Y_{p}$ see Ref. 66]. Possible measurements of other nuclei, like ${ }^{3} \mathrm{He}$, for which there is a single recent estimate, or ${ }^{6} \mathrm{Li}$, though difficult ${ }^{1}$, may greatly help in clarifying the overall soundness of Primordial Nucleosynthesis scenarios.

The main results of this paper are reported in Table 4 while our analysis can be summarized as follows:

i) we have described some key examples of the study performed to improve the accu-

\footnotetext{
${ }^{1}$ Actually, only for a smaller $\omega_{b}$, a large ${ }^{4} \mathrm{He}+D \rightarrow \gamma+{ }^{6} \mathrm{Li}$ reaction rate, at the upper limit of the present uncertainty range, and almost no depletion mechanism in act in hot and metal-poor PopII halo stars, one could reveal the primordial (as opposed to the cosmic-ray produced) ${ }^{6} \mathrm{~L} i$ in the near future.
} 
racy of the nuclear network adopted in our code. A full description will be presented elsewhere;

ii) we have performed a likelihood analysis of the WMAP results and BBN (Deuterium) abundance on $\omega_{b}$ and $N_{e f f}$, showing that the simplest standard BBN, with three neutrino as the only relativistic particles besides photons at the BBN epoch is largely consistent. In particular we found, at 95\% C.L. $\omega_{b}=0.023_{-0.002}^{+0.003}$ and $N_{\text {eff }}=3.6_{-2.3}^{+2.5}$. Notice the weak sensitivity of both $D$ and CMB data to $N_{\text {eff }}$. We stressed however that an improved accuracy of $D$ measurement may provide more accurate bounds on this parameter, independently of information on $Y_{p}$;

iii) we have studied, again via a likelihood method of the $X_{D}$ and $Y_{p}$ observables, the theory versus experiment status. We find that taking into account the present determination of ${ }^{4} \mathrm{He}$ shifts the preferred values of both $N_{\text {eff }}$ and $\omega_{b}$ towards smaller values than in the $C M B+D$ analysis, $\omega_{b}=0.021_{-0.004}^{+0.005}$ and $N_{e f f}=2.5_{-0.9}^{+1.1}$ at $95 \%$ C.L.. Finally we have also reported our estimate for ${ }^{3} \mathrm{He}$ fractional density and ${ }^{6} \mathrm{Li} /{ }^{7} \mathrm{Li}$ ratio (see Table 4 );

iv) in the framework of degenerate BBN we reported the bounds on possible extra relativistic degrees of freedom. Oscillations enforce the three active neutrinos to share the same small chemical potential at the BBN epoch and we find as a common bound $-0.03 \leq \xi_{e, \mu, \tau} \leq 0.11$ at $95 \%$ C.L. for $N_{\text {eff }}$ corresponding to three active neutrinos. In this case the agreement of ${ }^{4} \mathrm{He}$ theoretical expectation with experimental data improves. If we leave $N_{\text {eff }}$ as a free parameter we get $N_{\text {eff }}=3.9_{-3.4}^{+4.8}$ at 95\% C.L.. In particular the upper bound represents the largest extra contribution to the relativistic energy density due to exotic particles consistent with BBN.

After the huge progresses in the study and detection of Cosmic Microwave Background anisotropies, we think that further efforts in refining the BBN theoretical and experimental aspects may provide new pieces of information on cosmology and fundamental physics.

\section{Acknowledgements}

The authors are grateful to V.B. Belyaev, G. Imbriani, A. Melchiorri, S. Pastor, C. Rolfs, and F. Terrasi for useful discussions and valuable comments. 


\section{References}

[1] C.L. Bennett et al.(WMAP Coll.), Astrophys. J. Suppl. 148 (2003) 1.

[2] S. Esposito, G. Mangano, G. Miele, and O. Pisanti, JHEP 0009 (2000) 038.

[3] D.N. Spergel et al.(WMAP Coll.), Astrophys. J. Suppl. 148 (2003) 175.

[4] D. Kirkman, D. Tytler, N. Suzuki, J.M. O’Meara, and D. Lubin, astro-ph/0302006.

[5] D. Seckel, preprint BA-93-16, hep-ph/9305311 R.E. Lopez, M.S. Turner, and G. Gyuk, Phys. Rev. D56 (1997) 3191.

[6] R.E. Lopez and M.S. Turner, Phys. Rev. D59 (1999) 103502.

[7] S. Esposito, G. Mangano, G. Miele, and O. Pisanti, Phys. Rev. D58 (1998) 105023.

[8] S. Esposito, G. Mangano, G. Miele, and O. Pisanti, Nucl. Phys. B540 (1999) 3.

[9] S. Esposito, G. Mangano, G. Miele, and O. Pisanti, Nucl. Phys. B568 (2000) 421.

[10] L. Kawano, preprint FERMILAB-Pub-88/34-A; preprint FERMILAB-Pub-92/04-A.

[11] G. Fiorentini, E. Lisi, S. Sarkar, and F.L. Villante, Phys. Rev. D58 (1998) 063506.

[12] E. Vangioni-Flam, A. Coc, and M. Cassé, astro-ph/0002248, astro-ph/0009297; astro-ph/0101286.

[13] C. Angulo et al., Nucl. Phys. A656 (1999) 3. NACRE web site http://pntpm.ulb.ac.be/nacre.htm

[14] B. Zimmermann, W.A. Fowler, and G.R. Caughlan, Ann. Rev. A \&s A (1975).

[15] M.S. Smith, L.H. Kawano, and R.A. Malaney, Astrophys. J. Suppl. 85 (1993) 219.

[16] R.H. Cyburt, B.D. Fields, and K.A. Olive, New Astron. 6 (1996) 215.

[17] A. Cuoco, F. Iocco, G. Mangano, G. Miele, O. Pisanti, and P.D. Serpico, in preparation. 
[18] A.D. Dolgov, S.H. Hansen, and D.V. Semikoz, Nucl. Phys. B503 (1997) 426; addendum, Nucl. Phys. B543 (1999) 269.

[19] S. Esposito, G. Miele, S. Pastor, M. Peloso, and O. Pisanti, Nucl. Phys. B590 (2000) 539.

[20] G. Mangano, G. Miele, S. Pastor, and M. Peloso, Phys. Lett. B534 (2002) 8.

[21] S. Dodelson and M.S. Turner, Phys. Rev. D46 (1992) 3372.

[22] B.D. Fields, S. Dodelson, and M.S. Turner, Phys. Rev. D47 (1993) 4309.

[23] S.H. Hansen, G. Mangano, A. Melchiorri, G. Miele, and O. Pisanti, Phys. Rev. D65 (2002) 023511.

[24] P. Crotty, J. Lesgourgues, and S. Pastor, Phys. Rev. D67 (2003) 123005.

[25] E. Pierpaoli, Mon. Not. Roy. Astron. Soc. 342 (2003) L63.

[26] S. Hannestad, JCAP 0305 (2003) 004.

[27] R.H. Cyburt, B.D. Fields, and K.A. Olive, Phys. Lett. B567 (2003) 227.

[28] V. Barger et al., Phys. Lett. B566 (2003) 8.

[29] U. Seljak and M. Zaldarriaga, Astrophys. J. 469 (1996) 437.

[30] http://lambda.gsfc.nasa.gov

[31] L. Verde et al. (WMAP Coll.), Astrophys. J. Suppl. 148 (2003) 195.

[32] A. Kogut et al. (WMAP Coll.), Astrophys. J. Suppl. 148 (2003) 161.

[33] G. Hinshaw et al.(WMAP Coll.), Astrophys. J. Suppl. 148 (2003) 135.

[34] V. Barger et al., Phys. Lett. B569 (2003) 123.

[35] A.D. Dolgov, S.H. Hansen, S. Pastor, S.T. Petcov, G.G. Raffelt, and D.V. Semikoz, Nucl. Phys. B632 (2002) 363.

[36] Numerical Algorithm Group website: http://www.nag.co.uk 
[37] The Review of Particle Physics, Phys. Rev. D66 (2002) 010001.

[38] LUNA Collaboration, Nucl. Phys. A706 (2002) 203.

[39] C.R. Brune et al., Phys. Rev. C43 (1991) 875.

[40] EXFOR-CSISRS database, http://www-nds.iaea.or.at/exfor/

[41] G.M. Hale, D.C. Dodder, E.R. Siciliano e W.B. Wilson, ENDF/B-VI Evaluation Material 125, Revision 1 (1991).

[42] Bishop e al., Phys. Rev.80(1950) 211; Snell e al., Phys. Rev. 80 (1950) 637; T.S. Suzuki et al., Astrophys. J. 439 (1995) L59; Y. Nagai et al., Phys. Rev. C56 (1997) 3173; D. Cokinos and E. Melkonian, Phys. Rev. C15 (1977) 1636 and refs. therein.

[43] G. Rupak, Nucl. Phys. A678 (2000) 409.

[44] G.J. Schmidt et al., Phys. Rev. C56 (1997) 2565.

[45] G.M. Griffiths et al., Can J. Phys. 41 (1963) 724.

[46] G.J. Schmidt et al., Nucl. Phys. A607 (1996) 139.

[47] S.L. Blatt et al. Phys. Rev. 176 (1968) 1147.

[48] M. Fukugita, T. Kajino, Phys. Rev. D42 (1990) 4251.

[49] L. Page et al., astro-ph/0302220.

[50] B.E.J. Pagel, E.A. Simonson, R.J. Terlevich, and M. Edmunds, Mon. Not. Roy. Astron. Soc. 255 (1992) 325; E. Skillman and R.C. Kennicutt, Astrophys. J. 411 (1993) 655; E. Skillman, R.J. Terlevich, R.C. Kennicutt, D.R. Garnett, and E. Terlevich, Astrophys. J.431 (1994) 172; K.A. Olive and G. Steigman, Astrophys. J. Suppl. 97 (1995) 49.

[51] Y.I. Izotov and T.X. Thuan, Astrophys. J. 500 (1998) 188.

[52] Y.I. Izotov and T.X. Thuan, astro-ph/0310421 
[53] P. Bonifacio and P. Molaro, Mon. Not. Roy. Astron. Soc. 285 (1997) 847.

[54] S.G. Ryan, J.E. Norris, and T.C. Beers, Astrophys. J. 523 (1999) 654.

[55] P. Bonifacio et al., A\&̈A 390 (2002) 91B.

[56] P. Bonifacio, in The link between stars and cosmology, Eds. M. Chavez and D. Mayya, Dordrecht, Kluwer.

[57] P. Bonifacio, A\&A 395 (2002) 515B.

[58] T.M.Bania, R.T. Rood, and D.S. Balser, Nature 415 (2002) 54.

[59] E. Vangioni-Flam, M. Cassé e J. Adouze, Phys. Rep. 333 (2000) 365.

[60] K. Jedamzik, Planetary and Space Science 50 (2002) 1239J.

[61] W.H. Kinney and A. Riotto, Phys. Rev. Lett. 83 (1999) 366.

[62] J. Lesgourgues and S. Pastor, Phys. Rev. D60 (1999) 103521.

[63] J. Lesgourgues, S. Pastor, and S. Prunet, Phys. Rev. D62 (2000) 023001.

[64] J. Lesgourgues and M. Peloso, Phys. Rev. D62 (2000) 081301.

[65] M. Orito, T. Kajino, G.J. Mathews, and Y. Wang, Phys. Rev. D65 (2002) 123504.

[66] R. Trotta and S.H. Hansen, astro-ph/0306588. 